\title{
Encofrado perdido constituido por paja cohesionada con micelio como sustituto del poliestireno expandido
}

\author{
Formwork consisting of mycelium cohesionated straw \\ as a substitute of expanded polystyrene
}

J. D. Román-Ramos ${ }^{(*)}, \underline{\text { F. J. Luna-Molina }}{ }^{(*)}$, L. J. Bailón-Pérez ${ }^{(*)}$

RESUMEN

En esta publicación se presentan algunas de las cualidades físicas y mecánicas de un material constituido por micelio sobre sustrato a base de paja de trigo. El objetivo es aprovechar las bondades de las paredes celulares del hongo, compuestas por quitina, una sustancia orgánica muy abundante en la naturaleza y que presenta unas excelentes propiedades mecánicas que convierten este material en idóneo, no sólo para competir con los contaminantes plásticos tan tradicionalmente empleados en construcción, tales como el poliestireno, sino para sustituirlos, puesto que el compuesto es totalmente biodegradable. Los primeros ensayos a compresión simple efectuados sobre este conglomerado formado por el sustrato y la quitina arrojan resultados de resistencia mecánica prometedores.

Palabras clave: Hongos; micelio; resistencia; poliestireno (EPS); aplicaciones.

\section{ABSTRACT}

This paper presents some of the physical and mechanical properties of a material made of mycelium within wheat straw. The aim of this study is to take advantage of the excellent properties of the cellular walls of fungi, made of chitin, an organic substance very abundant in the nature that presents notable mechanical properties both to compete with very contaminant plastics usually used in construction, like expanded polystyrene, and to substitute them, as this new material is biodegradable too. First simple compression essays carried out on the mentioned conglomerate made of mycelium within wheat straw provide promising mechanical strength results.

Keywords: Fungi; mycelium; strength; polystyrene (EPS); applications.

(*) Universidad de Granada (España).

Persona de contacto/Corresponding author: jlunam@correo.ugr.es (F. J. Luna-Molina)

Cómo citar este artículo/Citation: Román-Ramos, J. D., Luna-Molina, F. J., Bailón-Pérez, L. J. (2014). Encofrado perdido constituido por paja cohesionada con micelio como sustituto del poliestireno expandido. Informes de la Construcción, 66(EXTRA-1): moo6, doi: http://dx.doi.org/10.3989/ic.13.097.

Licencia / License: Salvo indicación contraria, todos los contenidos de la edición electrónica de Informes de la Construcción se distribuyen bajo una licencia de uso y distribución Creative Commons Reconocimiento no Comercial 3.o. España (cc-by-nc). 


\section{INTRODUCCIÓN}

El material que se pretende sustituir, el poliestireno expandido (EPS, en adelante), ya fue clasificado en 1986 por la Agencia de Protección al Medioambiente Americana como el quinto producto químico cuya fabricación generaba más desechos peligrosos, utilizándose 1,5 litros de petróleo por cada $1 \mathrm{~m}^{3}$ de material. Incluso al final de su vida útil es contaminante, puesto que se descompone muy lentamente en los vertederos y ocupa un gran volumen de los mismos, por lo que normalmente se opta por incinerarlo.

En los últimos años, se han diseñado procesos menos contaminantes, como el uso de mezclas de residuos de EPS con escayola o yeso (1). Pero, aun así, esto no deja de ser una solución parcial, pues se sigue usando el EPS, y no se apuesta firmemente aún por estructuras de producción y materiales sostenibles. Un fuerte aliado en este desafío es la quitina, que es el segundo compuesto más abundante en la naturaleza tras la celulosa (2) (3) (4), pero que no es demasiado conocido.

La quitina es un polisacárido ( $\beta$-N-acetil-glucosamina), un polímero catiónico lineal, biodegradable, de alto peso molecular y con una resistencia a tracción muy superior a la de cualquier material natural (incluidos la fibra de carbono y el acero) (5). Su empleo produce un incremento del módulo de Young y de la rigidez de los materiales con los que se une (6) (7) (8) (9) y su presencia en la naturaleza es fundamental para la supervivencia de muchos seres vivos. Buen ejemplo de ello es que, por un lado, en forma de nanofibras con un módulo de Young de $150 \mathrm{GPa}$, compone la cutícula constituyente del exoesqueleto de los insectos, siendo un material ideal para que éstos realicen sus funciones vitales, ya que es muy eficiente y ligero (10). Por otro lado, en el reino de los hongos, la quitina es el componente principal de las estructuras de protección y resistencia (11) (12) (13) (14) (15), proporcionando la rigidez necesaria al micelio o estructura vegetativa. El micelio es la parte del hongo en la que se sustenta el estudio; se compone de hifas, estructuras cilíndricas y filiformes de 2 a 10 micrómetros de diámetro y hasta varios centímetros de longitud, y presenta un crecimiento medio de $1 \mathrm{~mm}$ por hora (16).

La otra parte fundamental del material es el sustrato. El producto que se propone podría conseguirse a base de diversos sustratos habitualmente empleados para cultivar hongos, tales como paja, aserrín, arroz, algodón, trigo, avena, cebada, soja, maíz y caña de azúcar, teniendo una aplicabilidad local en todo el planeta. En esta investigación se han utilizado como sustratos la paja y la turba, muy abundantes en España.

\section{OBJETIVOS}

Para llevar a cabo el estudio de este material, se han perseguido fundamentalmente tres objetivos.

El primer objetivo que se pretende es conocer las variables que afectan al crecimiento micelial.

Para ello, el material micelial a analizar se obtiene a partir del crecimiento del micelio sobre el sustrato. De este modo se consigue que cohesione toda la muestra, tanto fuera como dentro de los moldes que garantizan la forma de las probetas. Al final del proceso, se evalúa la resistencia a compresión simple del material.
Se analizan dos tipos de ensayos, uno sobre sustrato que ya ha sido agotado y otro sobre probetas formadas con micelio inoculado. Las probetas se someten a las condiciones ideales para el crecimiento del micelio estudiadas durante esta investigación, y son conservadas en laboratorio hasta que cohesiona el sustrato. Para este segundo tipo de material en estudio, el número de ensayos es mayor porque se pretende evaluar cómo influye la densidad del material, variable relacionada con la cohesión.

El segundo objetivo es comparar dos especies de hongos, Pleurotus ostreatus y Agaricus bisporus o champiñón común, cultivados en paja y turba respectivamente, evaluando cuál es la más idónea para conseguir un producto final con la mayor resistencia posible.

Por último, con el tercer objetivo se pretende comprobar si el material estudiado cumple algunos de los requisitos más relevantes para convertirse en un claro sustituto del EPS, esto es, exigencias fundamentalmente basadas en propiedades mecánicas y de la resistencia al fuego (17). A este efecto, se han estudiado varias aplicaciones, centrándose en su uso como encofrado perdido (18) (19) (20) (21).

\section{CAMPAÑA EXPERIMENTAL}

Para la realización de los ensayos se han elaborado cuatro familias de muestras, las tres primeras con la especie Pleurotus ostreatus y la última con Agaricus bisporus.

Para la confección de la «familia 1», se ha introducido el sustrato ya agotado en moldes que permiten la realización de probetas cúbicas de $15 \mathrm{~cm}$ de lado y se ha dejado crecer el micelio en torno a 21 días para que cohesione todo el volumen. En esta familia se distinguen dos tipos de probetas, la 1.1 y la 1.2 fabricadas con sustrato totalmente agotado y la 1.3, a base de sustrato no agotado y cuyo hongo presenta aún capacidad de desarrollo.

La «familia 2» de probetas se ha elaborado con sustrato muy poco agotado. En este caso, las probetas permanecieron los 5 primeros días en los moldes y, tras ello, se extrajeron de ellos y se introdujeron en bolsas de plástico perforadas para asegurar la entrada de aire hasta la edad de 28 días. Su procedencia es distinta a la de la familia 1. En esta familia se distinguen dos probetas, la 2.1, que ha sido tallada, es decir, cortada directamente de la paca con objeto de no romper la estructura del hongo, y la 2.2, que está compactada en dos tongadas.

Por otro lado, para la confección de la «familia 3», se realizaron probetas con sustrato inoculado no agotado, creciendo el micelio en laboratorio bajo unas condiciones ambientales controladas e idóneas para la expansión del micelio (22) (23) (24) (25) (26) (27) (28 (29), esto es, protegido de la luz, a $28{ }^{\circ} \mathrm{C}$ de temperatura y con una humedad del $80 \%$ durante 21 días. Se realizaron probetas con cinco densidades distintas con el fin de encontrar cuál sería la idónea. Se fabricaron tres probetas para cada densidad considerada: la 3.1, 3.2 y 3.3 de $450 \mathrm{~kg} / \mathrm{m}^{3}$; la $3.4,3.5$ y $3.6 \mathrm{de} 475 \mathrm{~kg} / \mathrm{m}^{3}$; la $3.7,3.8$ y $3.9 \mathrm{de}$ $500 \mathrm{~kg} / \mathrm{m}^{3}$, la 3.10 , 3.11 y 3.12 de $525 \mathrm{~kg} / \mathrm{m}^{3}$ y la $3.13,3.14$, 3.15 de $550 \mathrm{~kg} / \mathrm{m}^{3}$.

Por último, la «familia 4» se compone de una única probeta, realizada a base del sustrato agotado de Agaricus bisporus sobre turba, a diferencia también de las familias anteriores, confeccionadas sobre paja. Esta probeta se ha elaborado cortando el material con las medidas exactas del molde para in- 
troducirlo de una sola vez e intentar no destruir la estructura que el hongo pudiera ya haber creado.

\section{RESULTADOS DE LOS ENSAYOS REALIZADOS}

\subsection{Resultados de ensayos mecánicos}

Las cuatro familias de muestras han sido sometidas a ensayos de compresión simple, aplicando la carga en dos direcciones diferentes, atendiendo a lo que se pretendía determinar. Por un lado, se ha llevado a cabo la evaluación de la resistencia a compresión simple mediante la aplicación de la carga de compresión en la dirección paralela a la de compactación de las capas; por otro lado, para evaluar la cohesión que el mice-

Tabla 1. Valores de resistencia a compresión para una deformación del 10\% en los ensayos de compresión simple efectuados en la dirección paralela a la de compactación.

\begin{tabular}{|c|c|c|}
\hline Familia & Probeta & $\begin{array}{c}\text { Resistencia a compresión al 1o\% } \\
\text { de deformación (MPa) }\end{array}$ \\
\hline \multirow{2}{*}{1} & 1.2 & 0,139 \\
\hline \multirow{3}{*}{3} & 3.6 & 0,032 \\
\cline { 2 - 3 } & 3.9 & 0,042 \\
\cline { 2 - 3 } & 3.12 & 0,046 \\
\cline { 2 - 3 } & 3.15 & 0,054 \\
\hline
\end{tabular}

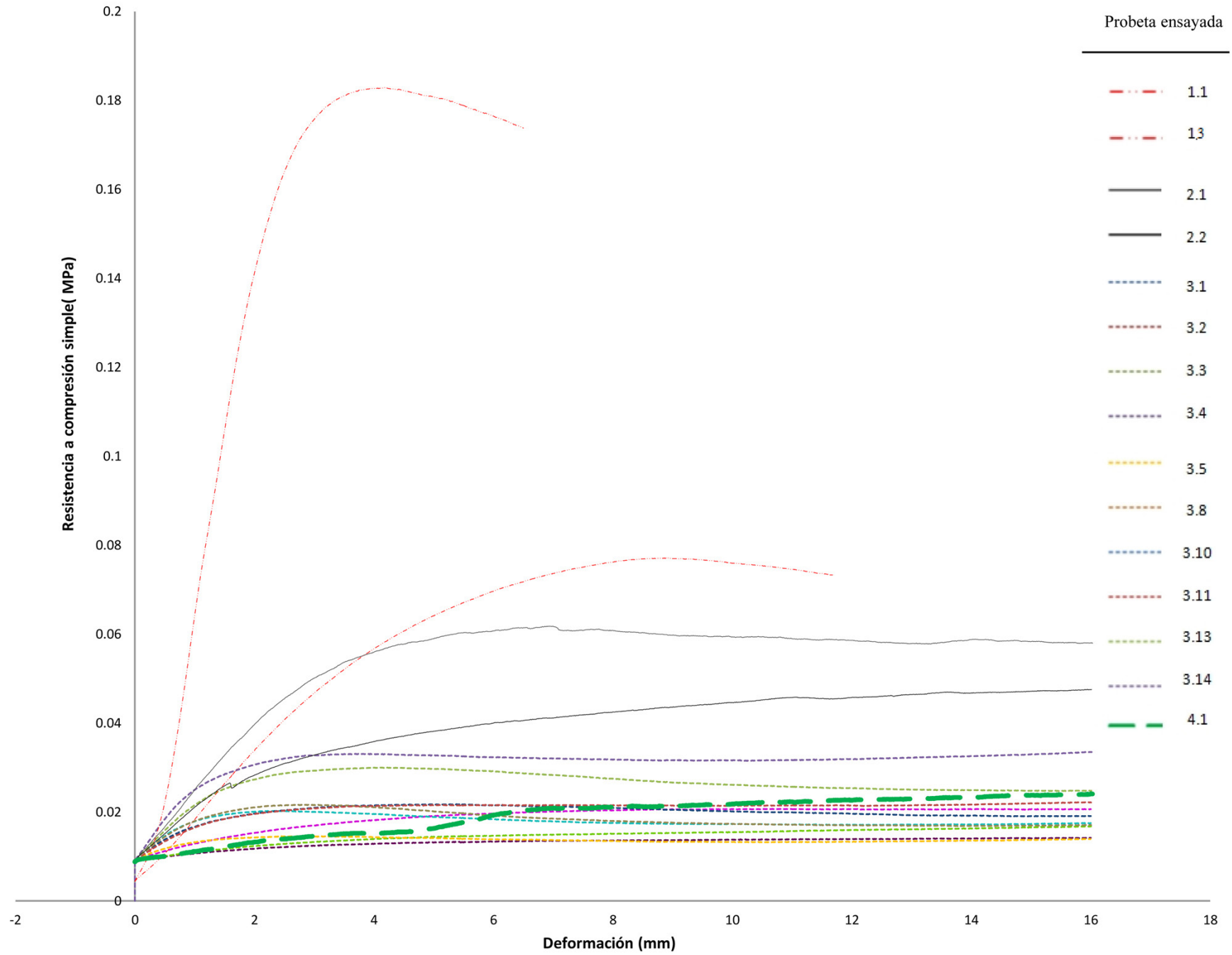

Figura 1. Curvas que relacionan la resistencia a compresión simple y la deformación de las probetas ensayadas en dirección perpendicular
Tabla 2. Valores de resistencia a compresión y de deformación alcanzados en rotura en los ensayos de compresión simple efectuados en la dirección perpendicular a la de compactación.

\begin{tabular}{|c|c|c|c|}
\hline Familia & Probeta & $\begin{array}{c}\text { Resistencia a } \\
\text { compresión en la } \\
\text { rotura (MPa) }\end{array}$ & $\begin{array}{c}\text { Deformación } \\
\text { en la rotura (\%) }\end{array}$ \\
\hline \multirow{3}{*}{1} & 1.1 & 0,191 & 3,9 \\
\cline { 2 - 4 } & 1.3 & 0,080 & 8,4 \\
\hline \multirow{3}{*}{2} & 2.1 & 0,062 & 6,9 \\
\cline { 2 - 4 } & 2.2 & 0,049 & 14,0 \\
\hline \multirow{4}{*}{3} & 3.1 & 0,015 & 6,2 \\
\cline { 2 - 4 } & 3.2 & 0,022 & 5,1 \\
\cline { 2 - 4 } & 3.3 & 0,016 & 10,3 \\
\cline { 2 - 4 } & 3.4 & 0,018 & 2,2 \\
\cline { 2 - 4 } & 3.5 & 0,014 & 4,2 \\
\cline { 2 - 4 } & 3.8 & 0,019 & 4,6 \\
\cline { 2 - 4 } & 3.10 & 0,023 & 2,2 \\
\cline { 2 - 4 } & 3.11 & 0,024 & 4,6 \\
\cline { 2 - 4 } & 3.13 & 0,026 & 4,1 \\
\hline \multirow{4}{*}{4} & 3.14 & 0,028 & 3,0 \\
\hline
\end{tabular}

lio aporta a la muestra, se ha aplicado la carga en la dirección perpendicular a la de compactación de las capas. Los resultados de estos ensayos se encuentran en las Tablas 1 y 2 y en la Figura 1. En la Figura 2, se presenta una probeta, pertene-

\footnotetext{
a la de compactación.
} 


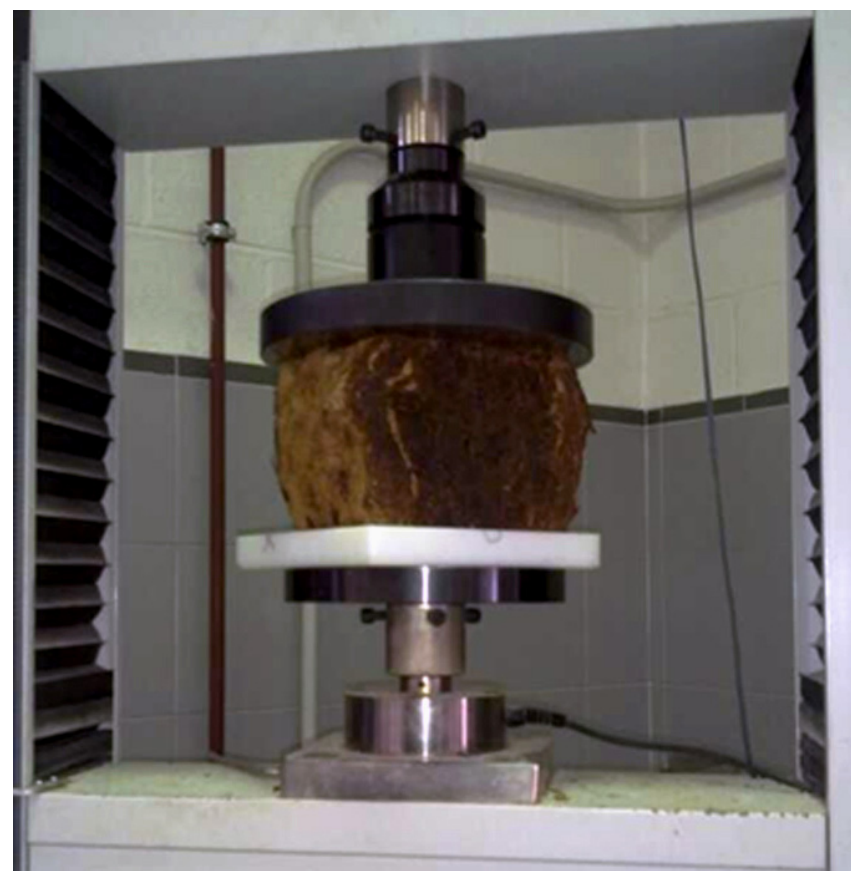

Figura 2. Probeta de la familia 1 después del ensayo.

ciente a la familia 1, justo después del ensayo a compresión simple en la dirección perpendicular.

\subsection{Resultados de los ensayos de resistencia ante el fuego}

En el presente estudio se ha querido también evaluar la resistencia ante el fuego del agregado sustrato-micelio.

En el Libro Blanco del EPS se puede leer «El EPS fundido normalmente no arde por chispas de soldadura o brasas de cigarrillos; sin embargo, las pequeñas llamas harán arder fácilmente al EPS de grado estándar a menos que contenga aditivos retardadores de la llama (EPS-SE)» (17).

El producto propuesto en esta investigación revoluciona este aspecto. Para comprobar si este material arde al contacto con una llama, se escoge, de entre todas las probetas confeccionadas, la que, a priori, parece ser la más desfavorable, es decir, la que tiene menos presencia de micelio, en concreto, resulta ser una probeta de la familia 3. Escogida la probeta, se le aplica una llama de soplete durante 20 segundos sobre una de las caras.

Se comprueba que la paja más superficial, seca y no envuelta en micelio, se consume. El fuego, al encontrar el micelio, no consigue prender el material, lo que revela que, sin necesidad de realizar ningún tratamiento superficial de la muestra, puede garantizarse que el material es prácticamente ignífugo, ya que el micelio actúa como una capa que resiste al fuego y lo extingue.

\section{DISCUSIÓN}

\subsection{Propiedades del material objeto de estudio}

\subsubsection{Aportación del micelio a la resistencia mecánica}

Tras estudiar la familia 1, se comprueba que la muestra 1.1, de sustrato agotado, soporta más carga que la muestra 1.3, de sustrato no agotado. Esto es debido a que la muestra de sustrato agotado posee más micelio. Igual ocurre cuando se comparan las familias 1 y 2; la familia 1 ha producido más cultivos y soportado más carga.

Respecto a la familia 3 se observa que, aun proporcionándose las condiciones idóneas para el crecimiento del micelio en laboratorio, debido al insuficiente tiempo de germinación, la resistencia que ofrece esta familia es menor que la de las familias 1 y 2, por lo que se deduce que la cantidad de micelio y, por tanto, el tiempo de crecimiento, es proporcional a la resistencia final obtenida a compresión simple.

\subsubsection{Isotropía del material}

La resistencia del material frente a una compresión en la dirección paralela a la de compactación, en general, es superior que si la compresión se realiza en dirección perpendicular. Sin embargo, si se comparan las probetas 1.1 y 1.2, ensayadas en dirección perpendicular y paralela respectivamente, se observa que la 1.1 aporta más resistencia que la 1.2, incluso con menor deformación. Este comportamiento es anómalo si se observan el resto de los casos en los que se compararon probetas ensayadas en direcciones perpendiculares. Puede ser debido a que, cuando la muestra 1.1 alcanzó una deformación del $15 \%$, su crecimiento fue exponencial, llegando a alcanzarse una resistencia de o,65 MPa para una deformación del $33 \%$, sin llegar a romper la probeta durante todo el ensayo, produciéndose un proceso de endurecimiento de la muestra para deformaciones superiores al $10 \%$.

Las familias 1 y 3 fueron fabricadas mediante aproximadamente 10 tongadas por probeta, con la consiguiente compactación tras la adición de cada capa, por lo que quizás haya sido el propio proceso de ejecución de las probetas el que haya hecho aumentar de forma tan significativa la resistencia a compresión en la dirección paralela a la compactación y disminuirla en la dirección perpendicular al modificar la estructura del micelio, proporcionando una prematura conclusión de que la resistencia de las muestras no es homogénea según la dirección de ensayo. Pero esta reflexión es totalmente errónea porque, si se analiza la probeta 2.1, realizada mediante el tallado, y la 2.2, compactada, puede concluirse que, al compactar, se rompe la estructura interna y la continuidad micelar puesto que la muestra tallada soporta más carga que la compactada.

\subsubsection{Influencia de la densidad en la resistencia a compresión obtenida}

Mediante el análisis de la familia 2 se comprueba que el material compactado presenta una resistencia un $26,5 \%$ mayor que el simplemente tallado, si bien es cierto que esto podría deberse simplemente a que su densidad es también superior.

Según los resultados de la familia 3, la probeta con mayor densidad proporcionó también más resistencia. En la figura 1 se observa como la curva de resistencia para esta familia se estabiliza al aumentar la densidad, por lo que puede concluirse que, de las densidades ensayadas, la ideal es $550 \mathrm{~kg} / \mathrm{m}^{3}$, si bien es cierto que deberían realizarse probetas con nuevas y diferentes densidades para comprobar hasta dónde mantienen esta correlación. 
5.1.4. Influencia del sustrato en la resistencia a compresión obtenida

El cultivo de Agaricus bisporus se realiza sobre turba y el de Pleurotus ostreatus sobre paja de trigo. Los resultados de la investigación arrojan que la resistencia del agregado formado tras inocular Pleurotus es mayor que la obtenida con Agaricus, aunque este último aglomerado muestra valores interesantes por los que merece la pena seguir estudiando.

\subsection{Comparación con el EPS}

En este apartado se evalúan si son admisibles los resultados obtenidos de los ensayos, teniendo en cuenta la aplicación a la que va a ser destinado el producto final. Para clarificar la discusión, se responde a continuación a dos preguntas clave, que se justifican con la normativa específica de ensayos para cada aplicación.

\subsection{1. ¿El material objeto de estudio cumple los valores mínimos de resistencia exigidos al EPS?}

Los campos de estudio donde el presente material puede ser un sustituto del EPS son la ingeniería civil y la edificación. Las principales aplicaciones del EPS en ingeniería civil son la fabricación de hormigón ligero, su utilización en barreras acústicas, su empleo para evitar daños por heladas en el pavimento, para crear planchas de drenaje, isletas de recreo y para controlar los asientos diferenciales en las plataformas de las carreteras. En cuanto a edificación, es conocido su uso como material aislante, en bovedillas para forjados, en ornamentación de fachada, en cerramiento de cubiertas y en aplicaciones tan novedosas como el sistema constructivo Nydion (30). Sin embargo, la aplicación más directa sería como encofrado perdido (31), molde empleado para dar forma al hormigón y que le confiere ciertas propiedades que lo mejoran como aislamiento y aligeramiento, puesto que presentan menos requerimientos para su comercialización.

No obstante, para poder emplear el material en las aplicaciones descritas, éste debe cumplir los requerimientos particulares de cada una. En este trabajo se ha querido comprobar si el material en estudio cumple los requisitos mecánicos de las cuatro aplicaciones más importantes, como son la de material de embalaje, el uso en carreteras para evitar asientos diferenciales, como aislante en edificación y en especial como encofrado perdido.

En la Figura 3 se observa que la familia 1 cumple sobradamente los requisitos de compresión simple a una deformación del 10\% exigidos al EPS para los cuatro usos anteriormente mencionados. Además, la familia 2 podría también utilizarse para tales fines puesto que su resistencia se encuadra entre la exigida al EPS que se emplea en carreteras y como material embalaje, que son las más exigentes. Las probetas de mayor densidad de la familia 3 y la familia 4 en su totalidad podrían usarse exclusivamente como encofrado perdido, si bien es cierto que el tiempo que ha tenido el micelio para crecer ha sido insuficiente y podría esperarse un aumento de la resistencia.

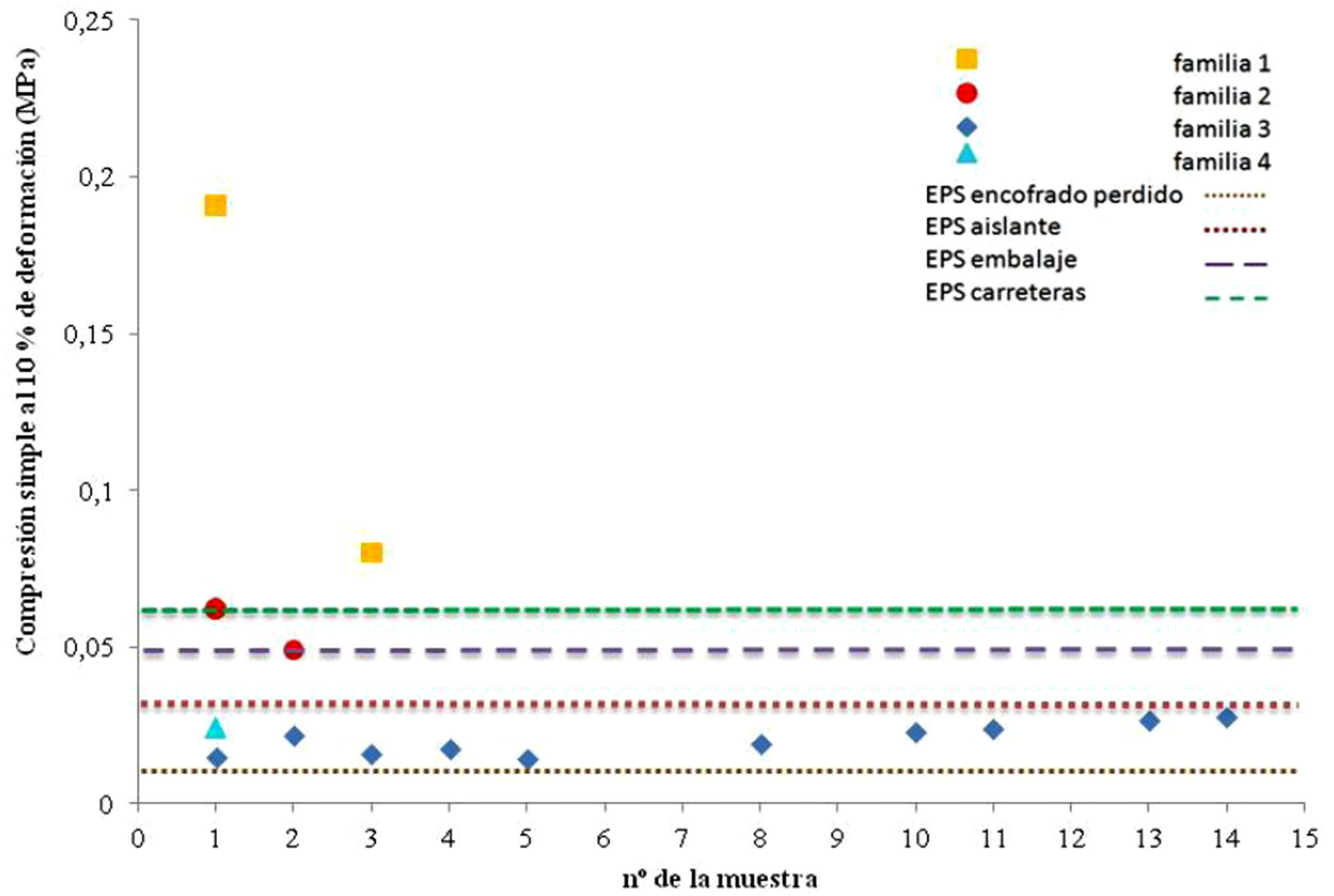

Figura 3. Comparación entre los resultados obtenidos en los ensayos realizados sobre el material objeto de estudio y los valores exigibles al EPS en las distintas aplicaciones consideradas. 
5.2.2. ¿El material objeto de estudio cumple los requerimientos ante fuego exigidos al EPS?

Se han realizado ensayos sobre el material a base de sustrato y micelio obtenido de la inoculación de Pleurotus ostreatus, de la familia 1 y 2.

En principio, la autoextinción que presenta el material al contacto con la llama es un resultado muy a tener en cuenta puesto que el EPS no resiste el calor de la misma. No obstante, el tipo de ensayo para evaluar la resistencia al fuego varía dependiendo de la aplicación a que vaya a ser destinado el producto.

\section{CONCLUSIONES}

Después del trabajo desarrollado en estos últimos meses, se ha llegado a conclusiones muy alentadoras que motivan el afán de seguir investigando con más detalle el material objeto de estudio. A continuación, se exponen las más importantes.

\subsection{Aportación del micelio a la resistencia mecánica}

La cantidad de micelio presente en el material y, por tanto, el tiempo de crecimiento del mismo, es proporcional a la resistencia a compresión simple.

\subsection{Isotropía del material}

Se puede concluir que este material es isótropo por la forma propia de crecimiento que experimenta el hongo en el interior de cualquier sustrato.

\subsection{Influencia de la densidad en la resistencia a compresión obtenida}

Las probetas con mayor densidad han arrojado valores de resistencia a compresión también más elevados, por lo que a priori parece que, a mayor densidad, mayor resistencia.

\subsection{Influencia del sustrato en la resistencia a compresión obtenida}

El análisis de la familia 4 lleva a la conclusión de que el rendimiento mecánico, medido a través del ensayo a compresión simple, del Agaricus en la turba es menor que el del Pleurotus en la paja.

\subsection{Sustitución del EPS}

Se concluye que el material a base de sustrato y micelio obtenido de la inoculación de Pleurotus ostreatus, de la familia 1 y 2, podría sustituir al EPS en las diversas aplicaciones estudiadas en cuanto a la resistencia mecánica y al comportamiento ante el fuego se refiere. La familias 3 y 4 cumplen los requisitos mecánicos para ser utilizadas como encofrado perdido pero no ocurre igual para el resto de aplicaciones estudiadas, aunque quizás haya podido deberse a que el tiempo que ha tenido el micelio para crecer ha sido insuficiente.

\subsection{Carácter biodegradable del material}

La utilización de material reciclado, no sólo plásticos sino productos de la industria del cemento, muy de moda en estos días (32), permite crear un circuito cerrado para una industria sostenible. En esta dirección, el material que aquí se presenta podría tener determinadas aplicaciones estructurales y, llegado el momento, podría ser no sólo devuelto al ciclo mediante una posible valorización del mismo, sino que podría dejarse consumir por sus características de biodegradabilidad.

\subsection{Comportamiento ante el fuego}

El agregado sustrato-micelio posee propiedades ignífugas como la resistencia a una llama de fuego y que el EPS no satisface, por lo que, a falta de ensayos específicos para la aplicación a la que finalmente vaya a ser destinado, el comportamiento de este producto ante el fuego es alentador.

\subsection{Resultado prometedor}

El producto debe cumplir una serie de exigencias particulares para cada uso. Los resultados de este estudio, si bien es cierto que basado en una campaña de ensayos no demasiado extensa, han demostrado que, de entre los requisitos exigibles a productos similares, como puede ser el EPS, el material objeto de estudio cumple los que a priori parecen más importantes. No obstante, se pretende seguir trabajando para corregir las debilidades de este material.

\section{FUTURAS LÍNEAS DE INVESTIGACIÓN}

Actualmente se está estudiando la influencia del tiempo y de la temperatura de secado de las probetas en la resistencia final con objeto de encontrar el secado ideal, es decir, aquel para el cual la resistencia de las probetas sea máxima.

Además, las futuras líneas de investigación que se pretenden acometer son estudiar si el material cumple todos los requerimientos para poder comercializarse en sus potenciales aplicaciones, comprobando en obra su correcto funcionamiento, detectando posibles fallos adicionales y proponiendo correcciones. Concretamente, para su uso como encofrado perdido, habría que estudiar la estabilidad dimensional, la absorción de agua, la imputrescibilidad y las propiedades termohigrométricas y térmicas.

\section{AGRADECIMIENTOS}

Este trabajo no hubiera sido posible sin la colaboración de la empresa Misur S.L. que nos ha cedido sus instalaciones y equipos. Agradecemos al profesor José Rodríguez Montero la absoluta disponibilidad de los medios disponibles en su laboratorio, al profesor Manuel Díaz y a los profesores Enrique Hernández y Luisa María Gil promotores de esta investigación. 


\section{REFERENCIAS}

(1) González-Madariaga, F. J. (2008). Mezclas de residuos de poliestireno expandido (EPS) conglomerados con yeso o escayola para su uso en la construcción. Informes de la Construcción, 60 (509): 35-43, doi: http://dx.doi.org/10.3989/ ic.2008.v60.i509.589.

(2) Ruiz-Herrera, J. (1993). La Quitina. El segundo compuesto orgánico más abundante en la Tierra. Investigación y Ciencia, (202): 42-49.

(3) Lárez-Velásquez, C. (2006). De Quitina y quitosano: materiales del pasado para el presente y el futuro. Avances en Química, 1(2): 15-21.

(4) Caprile, M. D. (2006). Obtención y Utilización de Quitina y Quitosano a partir de desechos de crustáceos. Ceiso: 1-6.

(5) Sánchez, A., Sibaja, M., Veja-Baudrit, J., Madrigal, S. (2007). Síntesis y caracterización de hidrogeles de quitosano obtenido a partir del camarón langostino con potenciales aplicaciones biomédicas. Revista iberoamericana de Polímeros, 8(4): 241-267.

(6) Ashby, M.F. (1999). Materials Selection in Mechanical Design, $2^{\text {nd }}$ ed. Oxford: Butterworth-Heinemann.

(7) Helge-Otto, F., Sachs, Ch., Romano-Triguero, P., Raabe, D. (2009). Influence of Structural Principles on the Mechanics of a Biological Fiber-Based Composite Material with Hierarchical Organization: The Exoskeleton of the Lobster Homarusamericanus. Advanced materials, 21(4): 391-400, doi: http://dx.doi.org/10.1002/adma.200801219.

(8) F.V. Vincent, J., G.K. Wegst, U. (2004). Design and mechanical properties of insect cuticle. Arthropod Structure \& Development, 33 (3): 187-199, doi: http://dx.doi.org/10.1016/j.asd.2004.05.006.

(9) Ogura, K., Kanamoto, T., Itoh, M., Miyashiro, H., Tanaka, K. (1980). Dynamic Mechanical Behavior of Chitin and Chitosan. Polymer Bulletin, 2(5): 301-304, doi: http://dx.doi.org/10.1007/BFo0266704.

(10) Franco-Chávez, K. G., Rodríguez-Navarro, S., Cervantes-Mayagoitia, J. F., Barranco-Florido, J.E. (2012). Enzimas y toxinas de hongos entomopatógenos, su aplicación potencial como insecticidas y fungicidas. Sociedades Rurales, Producción y Medio Ambiente, 12(23): 143-16o. http://148.206.107.15/biblioteca_digital/articulos/5-599-8595jkj.pdf.

(11) Pisabarro, A. G., Ramírez, L. (2006). VI Meeting on Genetics and Cellular Biology of Basidiomycetes. Universidad Pública de Navarra.

(12) Ruiz-Herrera, J. (2012). Fungal Cell Wall. Structure, Synthesis and Assembly, $2^{\text {nd }}$ ed. Boca Raton (Florida): CRC Press, Taylor \& Francis Inc.

(13) Ruiz-Herrera, J. (2005). Chitin. Biosynthesis in fungi. Sevilla: Universidad de Sevilla - Lex Scheffers.

(14) Ruiz-Herrera, J. (2005). La síntesis de la quitina en los hongos. Sevilla: Universidad de Sevilla-Lex Scheffers.

(15) Chang, S.-T., Miles, P.G. (2004). Mushrooms. Cultivations, nutritional value, medical effect and environmental impact. $2^{\text {nd }}$ ed. United States: CRC Press, Inc.

(16) Pérez, B. (2013). Identificación de hongos filamentosos con importancia fitopatógena. En Primer Congreso Internacional de Biotecnología UPAEP.

(17) EUMEPS. (2003). Libro Blanco del EPS. (pp. 1-60). http://www.anape.es/pdf/Libroblanco.pdf.

(18) Yoonseok, S., Taehoon, K., Hunhee, Ch., Kyung-In, K. (2012). A formwork method selection model based on boosted decision trees in tall building construction. Automation in Construction, 23: 47-54, doi: http://dx.doi.org/10.1016/j. autcon.2011.12.007.

(19) Metin, A., Osman, S., Serkan, S. (2005). Effects of formwork surface material son concrete lateral pressure. Construction and Building Materials, 19(4): 319-325, doi: http://dx.doi.org/10.1016/j.conbuildmat.2004.07.007.

(20) Perrot, A., Amziane, S., Ovarlez, G., Roussell, N. (2009). SCC formwork pressure: Influence of steel rebars. Cement and Concrete Research, 39(6): 524-528, doi: http://dx.doi.org/10.1016/j.cemconres.2009.03.002.

(21) Horvath, J. S. (1997). The Compressible Inclusion Function of EPS Geofoam. Geotextiles and Geomembranes, 15(1-3): 77-120, doi: http://dx.doi.org/10.1016/So266-1144(97)oooo8-3.

(22) Aranguren-Oroz, I. (2006). Ensayo de sustratos experimentales a base de residuos oleicos para el cultivo de hongos. Proyecto de Ingeniería Ambiental coordinado con Universidad de Florencia.

(23) Ardón-López, C. E. (2007). La producción de los hongos comestibles. Guatemala: Universidad de San Carlos de Guatemala. http://www.innovacion.gob.sv/inventa/attachments/article/2043/o7_1932.pdf.

(24) López, R. A. (1995). Cultivo de setas: alternativa alimenticia de la economía familiar (en línea). Veracruz, México: Universidad Veracruzana - Centro de Genética Forestal.

(25) Stamets, P. (2003). Growing gourmet and medicinal mushrooms. EE.UU: University of Arizona - Ten Speed Press.

(26) Staments, P. (2005). Mycelium running. EE.UU: University of Arizona - Ten Speed Press.

(27) Sánchez, J. E., Royse, D. (2001). La biología y el cultivo del pleurotus. México: Editorial Limusa, S.A.

(28) Shelley, E., Geoffrey, K. (2008). Hongos. Barcelona: Omega.

(29) Tellería, M. T. (2011). Los hongos. Madrid: Consejo Superior de Investigaciones Científicas.

(30) Palermo, M., Gil-Martín, L. M., Trombetti, T. Hernández-Montes, E. (2013). In-plane shear behavior of thin low reinforced concrete panels for earthquake re-construction. Materials and Structures, 46(5): 841-856, doi: http://dx.doi. org/10.1617/s11527-012-9937-8.

(31) Rodríguez-Santiago, J. (1990). Nuevas tendencias en estructuras de hormigón. Informes de la Construcción, 42 (410): 45-57, doi: http://dx.doi.org/10.3989/ic.1990.v42.i410.1417.

(32) Zaragoza, A. (2010). Circuito cerrado para una industria sostenible, aportaciones del sector cementero. Informes de la Construcción, 62(517): 53-59, doi: http://dx.doi.org/10.3989/ic.08.046. 